\title{
Self-Organized Profile Relaxation by Ion Temperature Gradient Instability in Toroidal Plasmas
}

\author{
Y. Kishimoto, ${ }^{*}$ T. Tajima, M.J. LeBrun, M.G. Gray, \\ J-Y. Kim, and W. Horton \\ Institute for Fusion Studies \\ The University of Texas at Austin \\ Austin, Texas 78712 \\ January 12, 1993
}

\begin{abstract}
roidal effects on the ion-temperature gradient mode are found to dictate the temperature evolution and the subsequent relaxed profile realization according to our toroidal particle simulation. Both in the strongly unstable fluid regime as well as in the near-marginal kinetic regime we observe that the plasma maintains an exponential temperature profile and forces the heat flux to be radially independent. The selforganized critical relaxed state is sustained slightly above the marginal stability, where the weak wave growth balances the wave decorrelation.
\end{abstract}

PACS Nos. 52.35.Kt, 52.55.Fa

"permanent address: Naka Fusion Research Establishment, Japan Atomic Energy Research Institute, Naka, Ibaraki 311-01, Japan

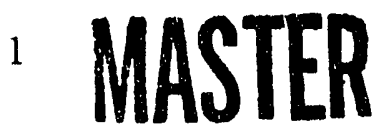


The ion temperature gradient (ITG) instability [1] (as well as the $\eta_{i}$ instability [2]) is believed to be a possible candidate respunsible for anomalous ion thermal transport including that during the high confinement $\mathrm{H}$-mode tokamak discharge, in which a steep ion temperature and flat density profiles are observed [3]. In the extreme limit, the ratio of the ion temperature gradient $L_{T}^{-1}$ to the density gradient $L_{n}^{-1}, \eta_{i} \equiv L_{n} / L_{T}$, becomes infinite and the linear stability property is then determined by the parameter $\epsilon_{T} \equiv L_{T} / R$, where $R$ is the major radius of the toroidal plasma, giving instability for $\epsilon_{T}<\epsilon_{T_{c}}$ where the critical $\epsilon_{T_{c}}$ is approximately $0.2-0.3$ [4]. Recent analyses of the DIII-D [5] and TFTR [6] plasmas indicate that although ITG signatures seem to be present, the temperature profile is close to marginal stability. A recent computational work in full toroidal geometry [7] shows radially extended nonlocal toroidal eigenmode (streamers) and radially increasing heat conductivity $\chi_{i}(r)$, consistent with experiments. These findings seem to challenge the currently held physical view that the anomalous transport is mainly the result of strong hydrodynamic turbulence of the tokamak plasma interior. In this Letter we investigate the nature of the temperature relaxation and heat transport and present an emerging new physical picture.

As we shall show below, the kinetic effects are important in the toroidal ITG mode. The perturbed circulating nonadiabatic ion distribution $\hat{f}_{i}^{n a}=\hat{g} \exp (i \mathcal{L})$ is obtained by kinetic analysis in ballooning space at $\theta=0$ (neglecting the radial dependence in amplitude), giving

$$
\hat{g}=\frac{e \hat{\phi}}{T_{i}} J_{0}(u) \frac{\Omega-\Omega_{*_{i}}^{T}\left(\tilde{v}_{\|}^{2}+\tilde{v}_{\perp}^{2}+\eta_{i}^{-1}-3 / 2\right)}{\Omega-\left(\tilde{v}_{\|}^{2}+\tilde{v}_{\perp}^{2} / 2\right)-\Omega_{t} \tilde{v}_{\|}} f_{M},
$$

and $\mathcal{L}=\mathbf{v} \times \mathbf{b} \cdot \mathbf{k}_{\perp} / \omega_{c i}(\mathbf{b} \equiv \mathbf{B} / B)$ for $\operatorname{Im} \omega>0$. In Eq. (1), $f_{M}$ represents the local Maxwellian, and $\Omega=\omega / \omega_{d}, \Omega_{*_{i}}^{T}=\omega_{*_{i}}^{T} / \omega_{d}, \Omega_{t}=k_{\|} v_{i} / \omega_{d}, u=k_{\theta} \rho_{i} \tilde{v}_{\perp}, \tilde{v}_{\|}=v_{\|} / v_{i}$, and $\tilde{v}_{\perp}=v_{\perp} / v_{i}\left(v_{i} \equiv\left(2 T_{i} / M_{i}\right)^{1 / 2}\right)$ where $\omega_{*_{i}}^{T}=-\left(c T_{i} / e B\right)\left(k_{\theta} / L_{T}\right)$, and $\omega_{d}=2 \epsilon_{T} \omega_{*_{i}}^{T}$ is magnetic drift frequency. Note that near marginal stability, the denominator in (1) approaches zero. In order to derive the dispersion relation in the entire complex $\omega$-plane, the velocity integrals must be carried out with analytical continuation from $\operatorname{Im} \omega>0$ to $\operatorname{Im} \omega<0$ across the 
marginal stability. In our simulation this crossing of the marginal stability is often observed so a careful treatment is necessary. By taking the zeroth velocity moment over (1), the nonadiabatic residual contribution to the ion density perturbation for the case $\operatorname{Im} \omega \leq 0$ and $\operatorname{Re} \omega>\omega_{b r} \equiv-k_{\|}^{2} v_{i}^{2} / 4 \omega_{d d}$ is given by

$$
\begin{gathered}
\delta \rho_{i, \mathrm{res}}^{n a}=-i \alpha \frac{k_{d i}^{2}}{\sqrt{\pi}} \hat{\phi}\left(\Omega+\frac{\Omega_{t}^{2}}{4}\right)^{1 / 2} \int_{-1}^{+1} d y \int_{0}^{+\infty} d t J_{0}^{2}(u) \exp \left(-\bar{v}_{\|}^{2}-2 t\right) . \\
\cdot\left[\Omega-\Omega_{*_{i}}^{T}\left(\bar{v}_{\|}^{2}+2 t+\eta_{i}^{-1}-3 / 2\right)\right] \delta\left[t-\left(\Omega+\frac{\Omega_{t}^{2}}{4}\right)\left(1-y^{2}\right)\right],
\end{gathered}
$$

where $\bar{v}_{\|}=\left(\Omega+\Omega_{t}^{2} / 4\right)^{1 / 2} y-\Omega_{t} / 2, u=\left(k_{\theta} \rho_{i}\right)(2 t)^{1 / 2}, k_{d i}^{2}=4 \pi n_{0} e^{2} / T_{i}$ and $\alpha$ is equal to $1 / 2$ for $\operatorname{Im} \omega=0$ and $\alpha=1$ for $\operatorname{Im} \omega<0$, respectively. In $(2)$ the resonant particles in torus form a ring in $\left(v_{\|}, v_{\perp}\right)$ space, instead of a strip as in the slab Landau resonance. Note that $\delta \rho_{i, \text { res }}^{n a}=0$ for $\operatorname{Re} \omega<\omega_{b r}$; there is a branch line along $\operatorname{Re} \omega=\omega_{b r}$ for $\operatorname{Im} \omega<0$. Using this expression, we coniputed the linear toroidal stability, shown in Fig. 1. Near the marginal stability for $X \equiv \Omega_{t}=0$, we find $\epsilon_{T_{c}} \sim 0.3$ and a nearly constant real frequency of $\operatorname{Re} \omega \sim 1.7 \omega_{d}$, which allows the thermal drift resonance.

We carried out a series of toroidal particle simulations of tokamak-like plasmas using the 'Toroidal Particle Code [7]. The toroidal and poloidal angles have mode numbers $n$ and $m$ respectively. Since the toroidal effects couple a particular poloidal mode $m$ to the $m+1$ and $m-1$ modes, a toroidal eigenmode tends to be nonlocal. The mode can extend radially from small $r$ and $m$ to large $r$ and $m$ through successive coupling from one rational surface to the next, for a given $n$ ( $n$ is a good quantum number for a tokamak). We take all successive $m$ 's ranging over the corresponding rational surfaces in the radial interval of interest for a given safety factor $q(r)$. In this case all successive $m$ modes may be coupled, while the $r$ dependence of $q(r)$ as well as $T_{i}(r)$ may break the toroidal coupling over a sufficiently wide radial distance. Although we ran both cases with multiple $n$ for multiple $m$ modes, and cases with a single $n$ for multiple $m$ modes, the observed results are qualitatively the same for reasons we will describe later. Thus in this Letter we concentrate on runs with 
a single $n$ and multiple $m$ 's $[n=9, m=5-16]$. We advanced ions with a full kinetic pusher, while electrons were treated adiabatically. Typical parameters are: the aspect ratio $R_{0} / a=4$, the average ratio of ion plasma frequency to ion cyclotron frequency $\left\langle\omega_{p i} / \omega_{c i}\right\rangle=1$, the average ratio of ion Larmor radius to minor radius $\left\langle\rho_{i} / a\right\rangle \simeq 0.02$, the magnetic shear parameter $\hat{s}=r q^{\prime} / q \sim 1.0$, the Debye length $\lambda_{D i} \approx \rho_{i}$, the ion temperature is given $\tan ^{-1}$ dependence, while the electron temperature and ion density were uniform at the start of the run. The initial values for $\epsilon_{T}$ ranges from 0.03 to much greater than 0.5 over the minor radius, thus the ITG modes should be excited in the fluid regime at the initial time.

Figure 2 shows the temporal evolution of the ion temperature (or its gradient $\epsilon_{T}^{-1}$ ) and the eigenfrequency of the toroidal ITG modes. The initial $\tan ^{-1}$ temperature profile relaxes quickly to a profile that fits well with an exponential function over a radial region $\left(\sim 15 \rho_{i}\right)$ [Fig. 2(a)] at time $t=2000-3000 \omega_{c i}^{-1}$ (time period I). By then the ITG instability has developed fully. In later times $\left(t=3600 \omega_{c i}^{-1}\right.$ in time period II and $t=6000$ in III) the slope of the temperature has become less steep but the functional dependence has remained exponential [Fig. 2(a)]. Around $t=3600$ the linear instability has saturated and the potential energy at its maximum. After $t=6000$ the profile hardly changed, although the wave activities do not subside. Shown in Fig. 2(b) is the measured frequency as a function of minor radius during the time period (III). The frequency shows one or two segmented steps (toroidal nonlocal eigenfrequencies) which globally obey the overall $\omega_{d}(r)$ curve. Each eigenmode thus extends over distance $15 \rho_{i}$ or so, not unlike that observed in [8], and then changes over to the other branch [9]. Corresponding to the time segments I, II, III, also shown in Fig. 2(d) is the eigenfrequency for $m=8$ as a function of time. The decreasing trend predicted of $\omega$ in I and a flat behavior in II are consistent with the linear theory in Fig. 1(b): $\epsilon_{T}$ approaches the marginal stability value $\epsilon_{T_{c}}$. The temporal evolution of temperature and frequency is closely related to that of the electric potential as shown in Fig. 3(a), where the time segments I, II, III correspond to the linear (growth) stage, the saturation stage, and the steady state. 
In time stage III we find that the fluctuations are far above the thermal level but steady in time.

The early potential structure in Fig. 3(b) shows the linear theoretical eigenfunction, displaying the ballooning signature [10]. In stage II the potential structure is well developed and shows a strong shearing motion, which eventually leads to tearing of $\mathbf{E} \times \mathbf{B}$ vortices and subsequently to reconnection of the torn vortices. The potential structure (particularly evident in phase II, but also in phases I and III) shows extended radial streamer whose poloidal shear is very pronounced in the toroidal interior $(\theta \sim \pi)$ and less so in the toroidal exterior $(\theta \sim 0)$. This may be interpreted as follows: the largest amplitude $(\theta \sim 0)$ due to the ballooning feature helps the strongest poloidal coupling take place at $\theta \sim 0$, leading to formation of the most prominent streamers, while at larger $\theta$ 's the poloidal differential rotation $\dot{\theta}=n \omega / q(r)$ gives rise to a strongly sheared radial structure of streamers. Such features were also observed in linear work [10].

Figures $3(\mathrm{e})$ and (f) shows the velocity distribution $f\left(v_{\|}, r\right)$ and $f\left(v_{\perp}, r\right)$ at stage III. Salient features in these figures are: (i) formation of clumps and/or holes in velocity distribution; (ii) asymmetric development in $v_{\|}$distribution; (iii) local cooling in $v_{\|}$distribution (note that such deviations from a Maxwellian distribution are impossible for a fluid model to reproduce). The clump/hole formation may be the result of mixing of hot and cold plasmas through nonlocal $\mathbf{E} \times \mathbf{B}$ convection during the ITG instability. Because of the clumpy nature of the ion distribution function, the estimated marginal stability condition (Fig. 1) predicted from (2) (where a Maxwellian was assumed) may change. The report in Ref. [5] that the DIII-D plasma seems "below" the marginal stability may be explained as clumps/holes enhanced damping mechanism so that the true marginal stability may have been lower than given by the Maxwellian theory.

Guided by observation described above, we are able to construct a phenomenological theory of temperature relaxation and heat transport. We have seen the ITG modes develop 
in three regimes: (I) the rapid, linear regime in which relaxation of temperature to an exponential profile results; (II) the quaslinear regime in which slow relaxation continues while maintaining the functional form of the temperature profile (exponential); (III) the relaxed regime in which the profile is nearly steady and kinetic processes dominate. Because of this time scale hierarchy we can expand the energy equation in terms of the time scale separation smallness parameter $\epsilon$, which is defined more concretely below. Let the time, temperature, and heat flux be expanded in the spirit of reductive perturbation theory as $\partial_{t}=\partial_{t_{0}}+\epsilon^{2} \partial_{t_{1}}+\epsilon^{4} \partial_{t_{2}} \cdots, T=T_{0}+\epsilon T_{1}+\cdots, Q=\epsilon Q_{0}+\epsilon^{2} Q_{1}+\cdots$, where $T_{0}$ means the self-similar exponential profile with $\partial T_{0} / \partial t_{0}=0$ and $t_{0}$ is the fastest time scale of the hydrodynamic instability evolution. For each order of $\epsilon$ we obtain an equation. Here we examine only the first two orders $\mathcal{O}(\epsilon)$ and $\mathcal{O}\left(\epsilon^{2}\right)$ :

$$
\begin{aligned}
& \frac{3}{2} n_{0} \frac{\partial T_{1}}{\partial t_{0}}+\nabla \cdot Q_{0}=0, \\
& \frac{3}{2} n_{0} \frac{\partial T_{0}}{\partial t_{1}}+\nabla \cdot Q_{1}=0
\end{aligned}
$$

where $Q_{0}=-\chi_{i}^{0} \nabla T_{0}, Q_{1}=-\chi_{i}^{0} \nabla T_{1}-\chi_{i}^{1} \nabla T_{0}$, and the source terms have been neglected, as they do not exist over the range of our present interest in our simulation.

If there is spatial dependence in $Q_{0}(r)$ in Eq. (3), it forces a rapid change in $T_{1}$ on the time scale $t_{0}$ so that it evolves to $\partial T_{1} / \partial t_{0}=0$, leading to $\boldsymbol{\nabla} \cdot \mathbf{Q}_{0}=0$ or $r Q_{0}(r)=$ const. This consequence is due to the strongly coupled ITG modes in nonlocal radial position in toroidal geometry, which have to either change the temperature profile [11] quickly and globally or to keep it steady. With our knowledge that the relaxed plasma has $T_{0}(r) \propto \exp \left(-r / L_{T}\right)$, and $r Q_{0}=$ const $=-r \chi_{i}^{0} \partial T_{0} / \partial r$, we are led to

$$
\chi_{i}^{0}=\frac{\text { const }}{r} \exp \left(+r / L_{T}\right)
$$

the universal radial dependence of the heat conductivity that increases as the minor radius increases, in agreement with most experiments. Equation (4) is the quasilinear evolution of 
$T_{0}$. By computing the second velocity rnoment of Eq. (1), we can calculate the heat flux:

$$
r Q=\operatorname{Re} \int d \mathbf{v}\left[\frac{1}{2} m v^{2}\left(\frac{c E^{*} \times B}{B^{2}}\right)\right] g \exp (i \mathcal{L})=\frac{n_{0} r k_{\theta} c e}{B}|\phi(r)|^{2} G\left(\Omega, \eta_{i}, L_{T}, r\right),
$$

where we are able to integrate the velocity integral $G$ (the normalized heat flux of the order of unity) and the resonant residual part of $G$ is given as

$$
\begin{gathered}
G=4 \sqrt{\pi} \alpha\left(\Omega+\frac{\Omega_{t}^{2}}{4}\right)^{1 / 2} \int_{-1}^{+1} d y \int_{0}^{+\infty} d t J_{0}^{2}(u)\left(\bar{v}_{\|}^{2}+2 t\right) \exp \left(-\bar{v}_{\|}^{2}-2 t\right) \\
\cdot\left[\Omega-\Omega_{\boldsymbol{*}_{i}}^{T}\left(\bar{v}_{\|}^{2}+2 a t+\eta_{i}^{-1}-3 / 2\right)\right] \cdot \delta\left[t-\left(\Omega+\frac{\Omega_{t}^{2}}{4}\right)\left(1-y^{2}\right)\right] .
\end{gathered}
$$

The above theory predicts that when $|\phi| \neq 0$ the heat flux $Q$ does not become zero even at $\operatorname{Im} \omega=0$ and remains positive well past critical $\epsilon_{T}$ [see Fig. 1(c)]. This is in sharp contrast to hydrodynamic turbulence theory [4]. In a special case $J_{0}^{2} \sim 1 \quad\left(k_{\theta} \rho_{i}<1\right), k_{\|} \sim 0$, the function $G$ becomes independent of $r$, as $\eta_{i}$ and/or $L_{T}$ are constant in the relaxed state, as we have found above [Fig. 2(a)]. The heat flux expression (6) then reduces to

$$
r Q(r)=\frac{n_{0} c e}{B} n q(r)|\phi(r)|^{2} G\left(\Omega, L_{T}\right),
$$

where $G$ is also shown in Fig. 1(c). So long as the safety factor $q(r)$ slowly changes in the spatial domain of our interest, as the theory requires $r Q(r)=$ const, the system forces $|\phi(r)|^{2}$ nearly constant. The $\delta|\phi(r)|^{2}=|\phi(r)|^{2}-\overline{|\phi|^{2}}$ contributes to the quasilinear evolution of $T_{0}$ in Eq. (4). While the radial dependence of $\chi_{i}$ is expressed in Eq. (5), the dependence of $\chi_{i}$ on plasma parameters are obtained from Eq. (8) as

$$
\chi_{i}=\left(k_{\theta} \rho_{i}\right) v_{i} L_{T}\left|\frac{e \phi}{T_{i}}\right|^{2} \operatorname{Re} G \propto \frac{T_{i}}{B} \frac{1}{R^{2}}
$$

where the level of $\phi$ is determined by the self-organized criticality condition, the details of which will be reported in a future publication. However, the basic idea is the following. As stated, the system is slightly above the marginal stability. In this state the growth of the waves $\left(\gamma_{\text {crit }}>0\right)$ and the decorrelation of waves have balanced and no profile change of the ion 
temperature results. On the other hand, if the system evolves in such a way that the plasma remains slightly unstable $\left(\gamma>\gamma_{\text {crit }}\right)$, then the transport changes the temperature profile quickly back to the criticality so as to be at $\gamma=\gamma_{\text {crit }}$. If the system becomes $\gamma<\gamma_{\text {crit }}$, then waves damp and transport decreases. The input power (or heat reservoir) then supplies the temperature near the core to rise to bring $\gamma$ to be at $\gamma_{\text {crit }}$. Thus, the $\gamma_{\text {crit }}$ may be determined by the balance equation $\gamma_{c r i t}-k^{2} D\left(|\phi|^{2}\right)=0$. In retrospect, the smallness parameter turns out to be $\epsilon=\left(\gamma_{\text {crit }} / \omega\right)^{1 / 2}$.

In conclusion we see through our toroidal particle simulation of the ITG (and $\eta_{i}$ ) modes (i) the toroidal geometry effect is important and results in a radially strongly coupled nonlocal modes that drive the temperature profile quickly until it reaches the relaxed state, as characterized above; (ii) the relaxed state is near (and slightly above) the marginal stability and the approximate global structure may be described by linear toroidal modes; (iii) since the mode is near marginal, the kinetic effects play an essential role and thus non-Maxwellian nature of velocity space prominently shows up; (iv) the experimental heat conductivity and other profile functionals [12] are considered to be the consequence of self-organized criticality.

The work was supported by the U.S. Department of Energy under contract \#DE-FG0580ET-53088, and JIFT. Discussions with M. Kotschenreuther, V. Wong, and S. Tokuda are gratefully appreciated. 


\section{References}

1. W. Horton, D.-I. Choi, and W.M. Tang, Phys. Fluids 24, 1077 (1981).

2. B. Coppi, M.N. Rosenbluth, and R.Z. Sagdeev, Phys. Fluids 10, 582 (1967).

3. K.H. Burrell and DIII-D Group, Phys. Rev. Lett. 59, 1432 (1987).

4. F. Romanelli, Phys. Fluids B 1, 1018 (1989); R.R. Domingues and R.E. Waltz, Phys. Fluids 31, 3147 (1988); R.R. Domingues and M.N. Rosenbluth, Nucl. Fusion 29, 844 (1989); W. Horton, B.G. Hong, and W.M. Tang, Phys. Fluids 31, 2971 (1988).

5. T.K. Kurki-Suonio, R.J. Groebner, and K.H. Burrell, Nucl. Fusion 32, 133 (1992).

6. M. Kotschenreuther, et al. (IAEA, Vienna, 1992) to be published.

7. M.J. LeBrun and T. Tajima, submitted to J. Comp. Phys.

8. M.C. Zarnstorff, et al. (IAEA, Vienna, 1992) to be published.

9. The linear dispersion analysis by assuming $k_{\|} \simeq 1 / 2 q R$ is indicating $\epsilon_{T} / \epsilon_{T c}>1$ (stabilizing) for $m \lesssim 8$ and $\epsilon_{T} / \epsilon_{T_{c}}<1$ (destabilizing) for $m \gtrsim 8$.

10. W.M. Tang and G. Rewoldt (to be published in Phys. Fluids).

11. S. Migliuolo, Phys. Fluids 28, 2778 (1985).

12. In $\eta_{i}$-mode case, we found that the functional form of the relaxed temperature profile is dictated by the density profile in such a way that the $\eta_{i}$ value becomes spatially independent, i.e. $\eta_{i}=$ constant. 


\section{Figure Captions}

1. Growth rate $\operatorname{Im} \omega / \omega_{d d}$ (a) and real part of the frequency $\operatorname{Re} \omega / \omega_{d}$ (b) versus $\epsilon_{T}$ for different value of $X=k_{\|} v_{i} / \omega_{d}$. Figure (c) shows the growth rate $\gamma R / v_{i}$ (solid line) and normalized heat flux $G=\bar{Q} \cdot B / r_{0} m c e|\phi|^{2}$ (dashed line) versus $k_{\theta} \rho_{i} / \sqrt{2}\left(\equiv k_{\theta} \bar{\rho}_{i}\right)$.

2. Temporal evolution of the ion temperature at three time steps (a) and the measured real frequency $\omega / \omega_{c i}$ (b) as a function of $r / a$ during time period III $\left(t=6000-12000 \omega_{c i}^{-1}\right)$. Dashed line represents $\omega_{d}(r) / \omega_{c i}$. Time evolution of measured $\epsilon_{T}(\mathrm{c})$ and real frequency $\omega / \omega_{d}(\mathrm{~d})$ are also shown.

3. Time evolution of the potential field for $m=8$ (a) and putential contour at each time segments I, II, and III ((b)-(d)). Solid and dashed lines represent plus and minus sign of the potential. (e)-(f) Contour of ion velocity distribution at $t=6000 \omega_{c i}^{-1}$. 

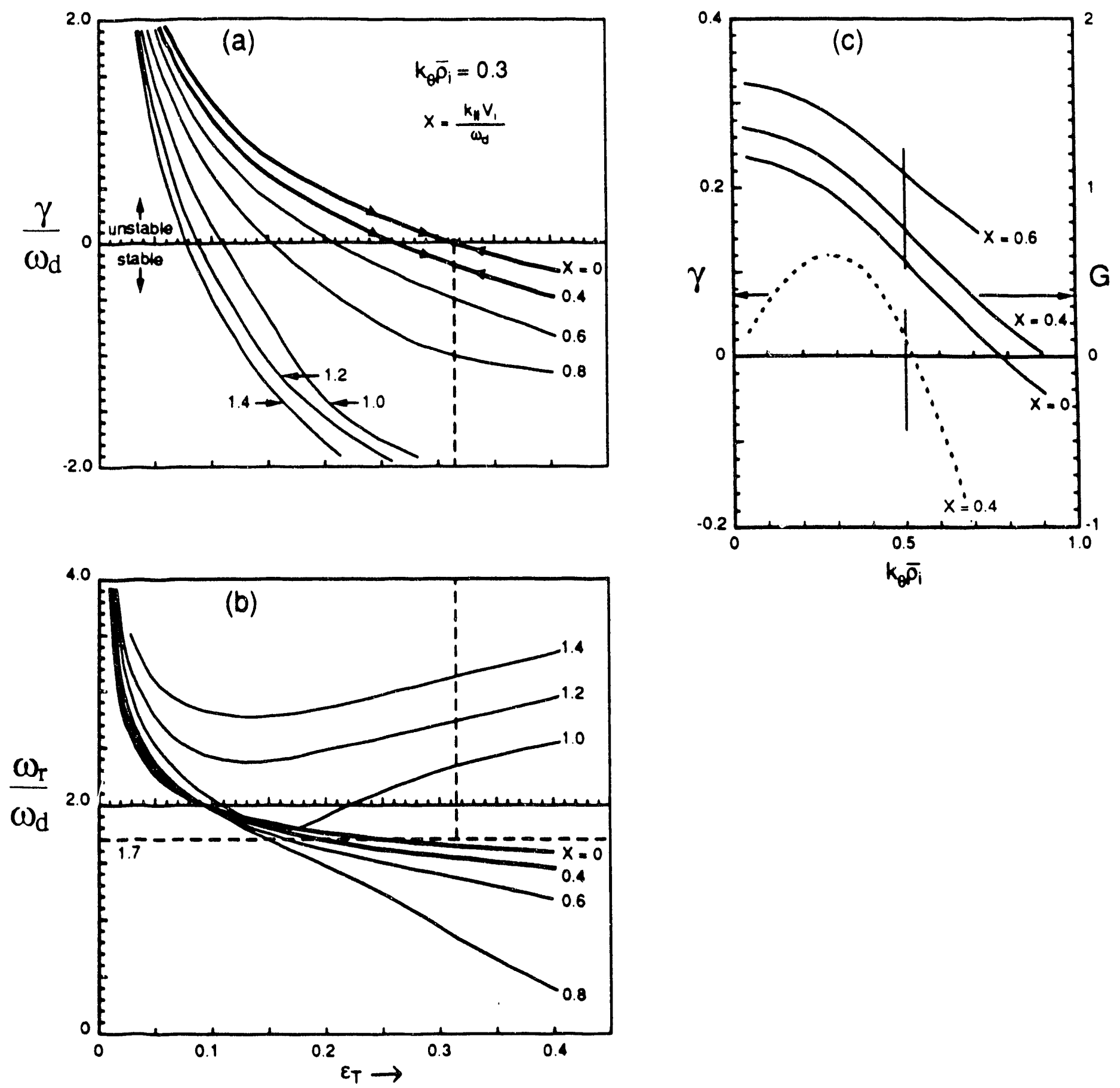

Fig. 1 

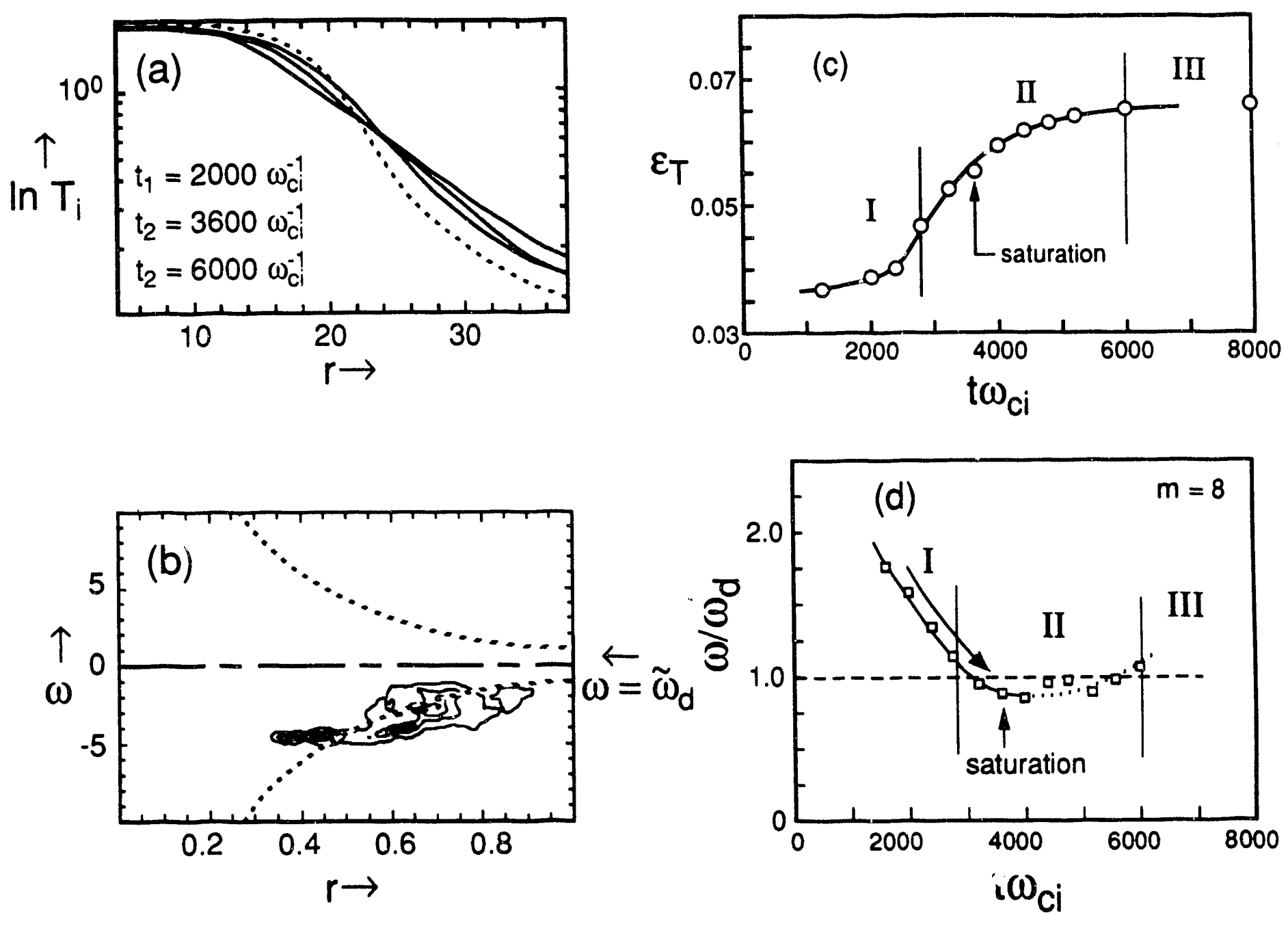

Fig. 2 
(a)
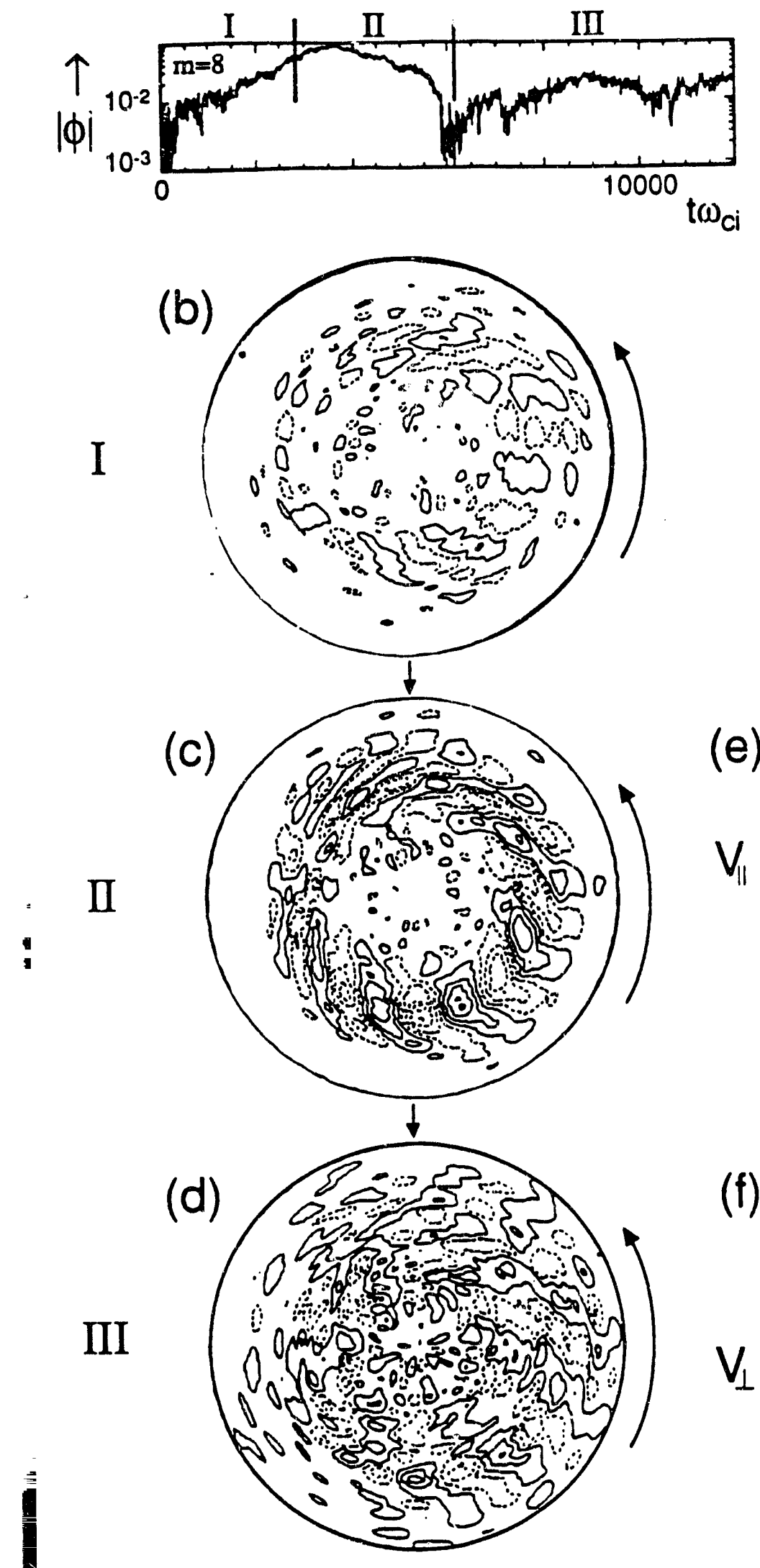

(e)

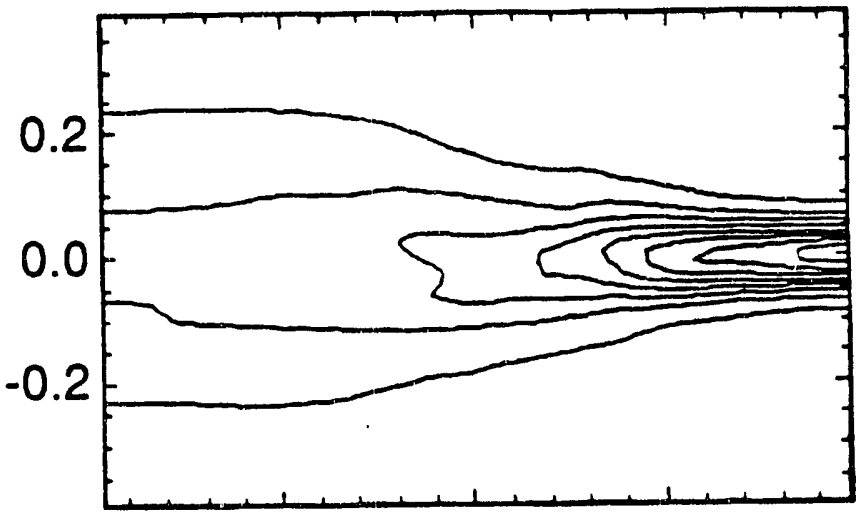

(f)

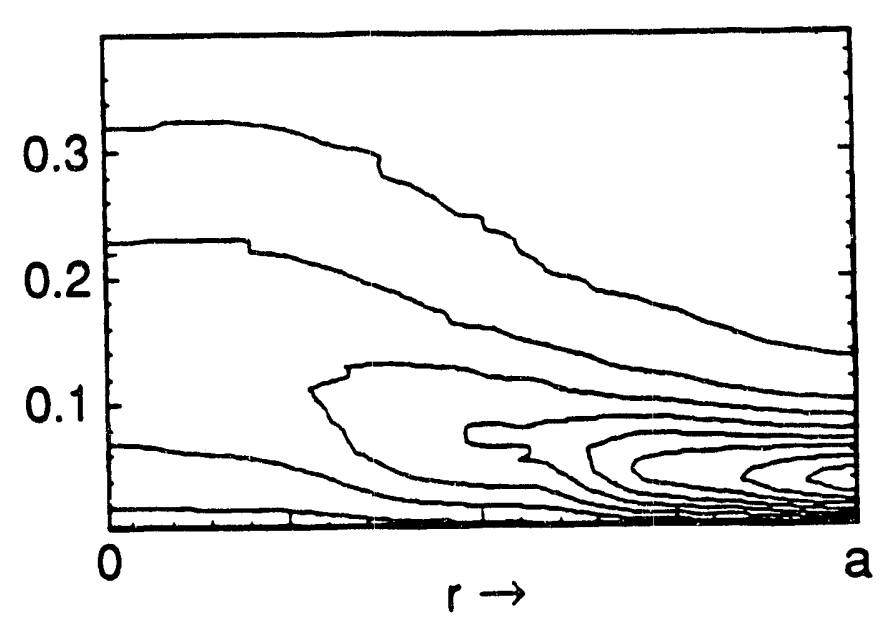

Fig. 3 

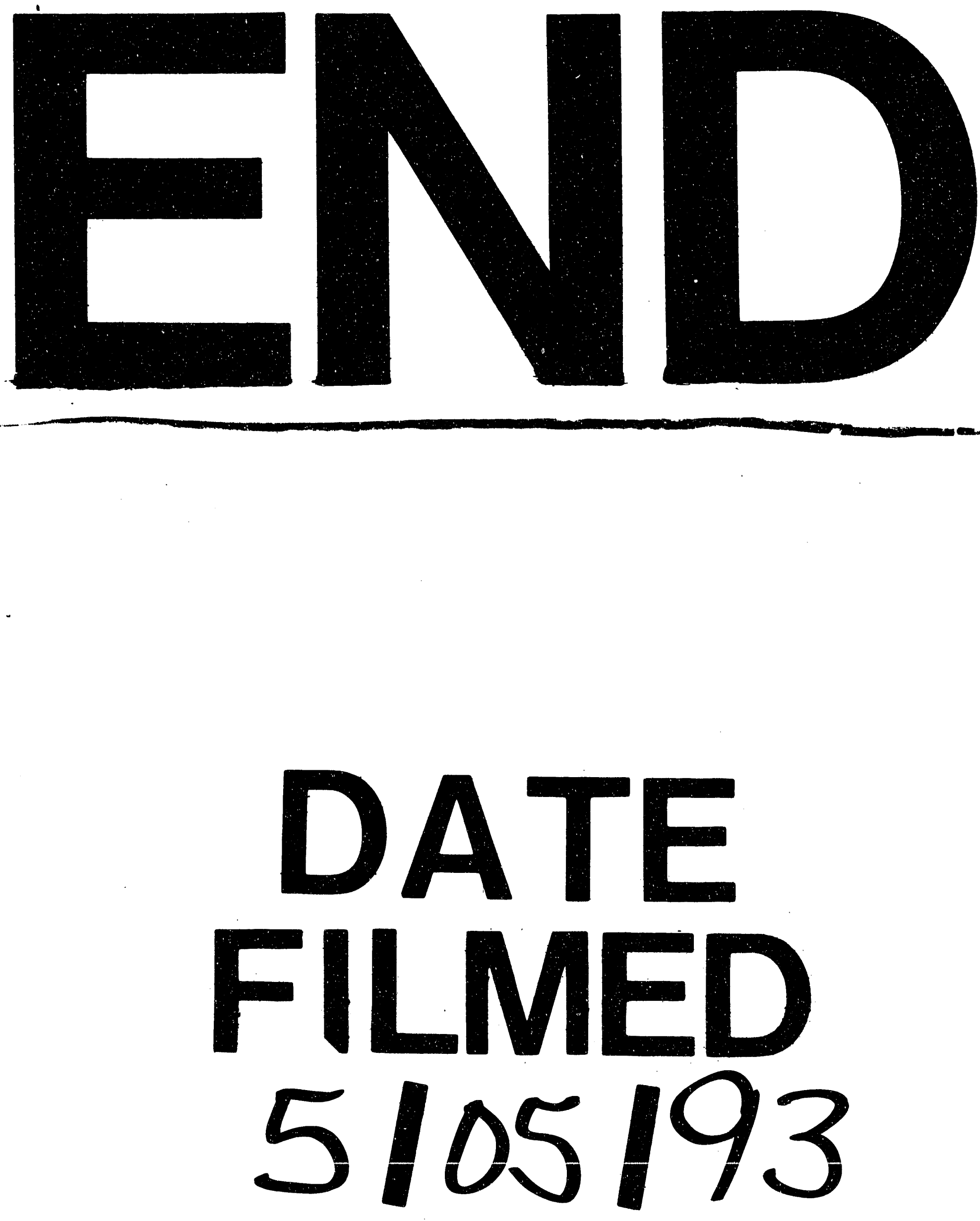


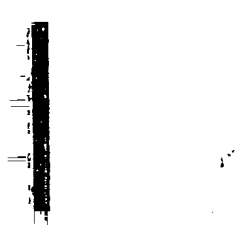

